Questions de communication

$24 \mid 2013$

Renouvellement des mises en scène télévisuelles de la politique

\title{
François FARCY, Jean-François GAYRAUD, Le
} renseignement criminel

Paris, CNRS Éd., coll. Arès, 2011, 132 pages

\section{Gérald Arboit}

\section{OpenEdition}

\section{Journals}

Édition électronique

URL : http://journals.openedition.org/questionsdecommunication/8796

DOI : 10.4000/questionsdecommunication.8796

ISSN : 2259-8901

\section{Éditeur}

Presses universitaires de Lorraine

\section{Édition imprimée}

Date de publication : 31 décembre 2013

Pagination : 280-282

ISBN : 978-2-8143-0182-5

ISSN : $1633-5961$

\section{Référence électronique}

Gérald Arboit, "François FARCY, Jean-François gaYraud, Le renseignement criminel », Questions de communication [En ligne], 24 | 2013, mis en ligne le 01 février 2014, consulté le 22 septembre 2020. URL : http://journals.openedition.org/questionsdecommunication/8796 ; DOI : https://doi.org/ 10.4000/questionsdecommunication.8796 
connaissances étant une procédure de gestion très spécifique, elle demande à chaque entreprise une grande souplesse managériale et une grande capacité d'adaptation. Dans le troisième chapitre (pp. 30-38), l'auteure explique que les entreprises faisant le pari de centrer leurs activités autour de la connaissance sont obligées de réinventer de nouvelles modalités organisationnelles : « Le concept de l'entreprise centrée connaissance (knowledge-centric enterprise) aussi appelé organisation 2.0 s'est développé, [...] inspiré d'analyses théoriques de l'évolution de la gestion des connaissances et des organisations, mais aussi d'analyses de choix stratégiques et structurels de certaines entreprises »(p. 69). Ensuite, l'auteur prend l'exemple de la société Google qui, par son positionnement professionnel, valorise la connaissance de ses salariés qui sont tous, sans exception, considérés comme détenteurs de connaissances inestimables pour l'entreprise. Cela renforce encore la légende qui veut que, « chez Google, l'efficacité de l'entreprise repose sur l'ambition, la créativité, la liberté, le bien-être des salariés, la satisfaction du client et l'éthique » (p. 78). Ce sentiment d'appartenance est encouragé et recherché par les recruteurs de la société américaine qui vérifient scrupuleusement que leurs futurs salariés seront en totale adéquation avec le mythe et la culture Google.

Dans la conclusion de l'ouvrage (pp. 99-108), Aurélie Dudezert insiste sur le fait que l'émergence de la ressource connaissance dans les organisations - ressource qui n'est pas un phénomène naturel, mais un construit social - remet au cœur de l'organisation la question de l'action collective et la dimension sociale de la technologie. C'est là l'apport considérable de ce livre qui intéressera tous ceux (étudiants, futurs salariés, managers, chercheurs, etc.) qui s'intéressent de près aux mutations organisationnelles.

\section{Alexandre Eyries \\ 13M, université Nice Sophia Antipolis, F-06200 alex.eyries@yahoo.fr}

\section{François FARCY, Jean-François GAYRAUD, Le renseignement criminel.}

Paris, cnRs Éd., coll. Arès, 201 I, I 32 p.

$\|$ est des livres comme des régimes qualifiables d'« Ancien régime ». Celui de François Farcy et JeanFrançois Gayraud appartient à cette catégorie tant on comprend qu'il est spécialement écrit pour nourrir le corpus scientifique de la contestée $75^{\mathrm{e}}$ section du Conseil national des universités de « criminologie », créée le 13 février et supprimée le 6 août 2012. L'entreprise de légitimation est doublement signée : une publication dans la collection dirigée par Xavier Raufer et une préface rédigée par Alain Bauer (pp. 9-II). Ce dernier y vante ce « nouveau paradigme » (p. 10) que serait le renseignement, d'un point de vue analytique autant qu'opérationnel, pour la police. Depuis les séminaires de l'amiral Pierre Lacoste, au milieu des années 90, et notamment les constats de Jean-Marc Berlière (Le renseignement à la française, Paris, Éd. Economica, 1998, pp. 9-27, ici pp. 13-20), il est admis que la police, comme les douanes, participent du renseignement de sécurité et font preuve, autant que faire se peut, d'anticipation et de pro-activité.

Cela dit, le cours ouvrage des deux commissaires belge (François Farcy) et français (Jean-François Gayraud) se décline comme une dissertation d'étudiants... en « criminologie ».Après une longue introduction (pp. 1 3-24) destinée à présenter l'« approche géopolitique et globale des études criminelles » chère à Alain Bauer et Xavier Raufer (etYves Roucaute, «Une vocation nouvelle pour la criminologie », Sécurité globale, 5, automne 2008, pp.89-93, ici p. 9|), s'enchaînent deux chapitres déséquilibrés, présentant le renseignement et son acception criminelle - «Qu'est-ce que le renseignement criminel et à quoi sert-il ? » (pp. 25-64) - et expliquant comment en faire - « Comment fait-on du renseignement criminel » (pp. 65-93). Logiquement, une courte conclusion - « Contre la résilience criminelle, la profondeur du renseignement d'intérêt criminel » (pp. 95-96) - rappelant que le « renseignement inscrit l'activité policière dans le qualitatif et le moyen terme ("l'efficacité"), non dans le quantitatif et le court terme » (p. 95) vient clore la démonstration et ouvre sur une suite de quatre annexes présentant l'état actuel du renseignement criminel dans le monde anglo-saxon (pp. 99-102), en France (pp. 103109), en Belgique (pp. I | | - 125) et un cas pratique tiré de l'actualité des subprimes (pp. 127-128).

L'objectif affirmé du livre est de « proposer un nouvel outil » (p. 23) aux décideurs politiques et administratifs, à savoir le « renseignement criminel, une notion encore balbutiante » (p. 2l). Pour l'atteindre, les auteurs mobilisent les expériences anglo-saxonnes de community policing (« police de proximité ») et d'intelligence led policing (« fonction policière guidée par l'information $»)$, remontant aux années 70-80, mais surtout de l'expérience belge, élaborée empiriquement suite aux différentes affaires judiciaro-médiatiques (commandant François, 1980 ; Rebelle, 1992-1996 ; Dutroux, 1996-1998 ; Brabant Wallon, 1997) et une adaptation à l'environnement international (trafic de drogue, crimes organisés turc et kurde, terrorisme) (pp. 79-93). Toutefois, 
cette polyvalence des menaces intérieures, dont les premiers signes apparaissent en Europe dès la fin des années 60, constitue pour les auteurs « la globalisation la plus aboutie depuis la fin de la guerre froide » (p. 19). Selon eux, « la prise de conscience de l'importance du "réel criminel" dans nos société est si lente » que la transnationalisation du crime est devenu un phénomène « massif [...], enraciné territorialement $[\ldots]$, organisé $[\ldots]$, résilient $[\ldots]$, destructeur $[\ldots]$, ou encore hybride $[\ldots]$, corrupteur $\gg($ p. 20). Chose que le crime a toujours été, comme le montre la société européenne de l'entre-deux-guerres où les criminels, jamais réellement solitaires si l'on compte les réseaux de soutien qu'ils utilisaient, se jouaient déjà des frontières. Le même postulat de nouveauté conféré au renseignement criminel (pp. |8-2I) apparait aux yeux des recherches récentes comme largement anhistorique (Frédéric Lemieux, Normes et pratiques en matière de renseignement criminel : une comparaison internationale, Québec, Presses de l'université Laval, 2006 ; pour une mise en perspective historique, Hélène L'Heuillet, Basse politique, haute police. Une approche historique et philosophique de la police, Paris, Fayard, 200 I ; Jean-Marie Berlière, « Police et renseignement », pp. 9-27, in : Pierre Amiral, Pierre Lacoste, dirs, Le Renseignement à la française, Paris, Economica, 1998 ; Gérald Arboit, Des services secrets pour la France, Paris, cNRs Éd. 2014). Mais, les auteurs tiennent trop à provoquer un « choc culturel» (p. 23) pour s'en soucier. Ils cherchent - et l'on ne les en blâme pas - à extirper « le concept même de "renseignement" [...] du monde du secret (des "services secrets/spéciaux") afin de pouvoir devenir un principe de management » (p. 23). Or, définir le renseignement est toujours difficile, surtout si on doit le rendre intelligible au grand public - ce à quoi, d'évidence, le livre ne s'attèle pas -, et les auteurs recourent à un habillage souvent habile, comme celui contestant la définition totalisante de Carl von Clausewitz - puisée dans l'Espion d'Alain Dewerpe (Paris, Gallimard, 1994) plutôt que dans le texte original où le penseur prussien se montre plus péjoratif à l'égard du renseignement - pour en arriver à paraphraser Simone de Beauvoir : « On ne "nait" pas renseignement, on le devient » (p. 27). Soit ! Lorsqu'ils se contentent de cerner cet objet spécifique, les deux policiers lui apportent pourtant une lecture intelligente. Le secret en est réduit à sa dimension résultante plutôt qu'à « une donnée a priori »(p. 28), tandis que le renseignement est présenté comme un cycle - recherche (pp. 42-50), analyse (pp. 50-57), diffusion stratégique aux décideurs politiques et tactique aux services de police (p. 59) - et non comme une rareté de l'information ou d'une émotion de l'opinion publique (pp. 29-30). De cette «notion si abstraite de "renseignement" » (p. 35), les auteurs tirent une « conception étroite, le renseignement criminel [qui] répond strictement à l'objet de police judiciaire » (p. 37). Cette construction du renseignement de sécurité, ainsi qu'on le qualifie généralement, semble prendre modèle sur le « Renseignement d'intérêt militaire » élaboré au milieu des années 90 en France afin d'élargir le strict concept militaire, c'est-à-dire de champ de bataille, pour en faire un outil de décision politico-militaire convenant mieux au nouveau concept opérationnel post-Guerre froide. De ce point de vue, la nouveauté paradigmatique contenue dans l'ouvrage apparait plus intelligible, tant « les services de police ont par tradition une habitude à s'orienter eux-mêmes » (p. 52). Or, en matière de renseignement, l'orientation, c'est-à-dire l'action consistant à indiquer aux différents services de recherche les informations à rapporter, les lieux où les trouver et les conditions dans lesquelles ils peuvent agir, est une étape fondamentale. Pour les auteurs, cette tradition policière serait le « trait commun et banal à la sous-culture policière », tant « il est sain en démocratie que les choix fondamentaux de politique anticriminelle relèvent explicitement du pouvoir politique et non subrepticement de l'administration » (p. 52). En démocratie, ces choix ne s'expriment-ils pas par la loi, qui constitue une orientation importante?

Les auteurs connaissent trop le fonctionnement de la police pour croire réellement ce qu'lls écrivent, conscients que «l'information existe mais la bureaucratie ignore qu'elle sait » (p. 59). Aussi se demandent-ils si ce nouveau paradigme ne serait autre chose qu'un 《 réflexe professionnel à généraliser ? 》. Le livre dérive alors du concept prétendument novateur du renseignement vers d'autres questions, celle du « management et de l'organisation administrative » (p. 59) d'abord, celle de la politique anticriminelle ensuite. Dans un cas, ils plaident pour une meilleur " implication sur les modes de fonctionnement (gestion, management) et de ce fait sur la "culture" des services » (p. 102). Dans l'autre, ils se demandent « qui $[\ldots]$ souhaite savoir réellement la totalité de la vérité? Le public peut-être, les autorités publiques, plus épisodiquement » (p. 64). À cet aveuglement plus ou moins voulu des décideurs s'ajoutent « des obstacles financiers » (p. 72). Les auteurs semblent ne pas vouloir comprendre que le principal problème est la nécessité du partage de l'information au-delà des logiques de service, occasionnant ce qu'ils nomment justement des « guerres des polices » (p. 83). La grande leçon du I I septembre 200 I, dont ils usent et abusent comme 
exemple, est justement cette difficulté à reconnaitre l'information utile et à bien la faire remonter vers ceux qui en ont réellement besoin.

Puis, ressortentles clivages entre « cousins 》, ces « services intérieurs et extérieurs, civils et militaires » (p. 69) qui se complairaient encore trop dans « leurs domaines habituels (espionnages et terrorisme) » (p. 69) pour se soucier de la criminalité transnationale, alors qu'elle est devenue leur priorité depuis les années 80 au moins. Mais il est vrai que la vision du renseignement, au niveau de «l'ilotier ou agent de quartier, figure centrale d'une police de renseignement » (p. 7I), dont la finalité reste l'arrestation, peut sembler incompatible avec une finalité tout autre, résidant dans l'identification des réseaux et la pénétration des filières pour les démanteler. Et pas nécessairement les juger! Cette incompréhension se trouve contenue dans l'expression « cadre légal »(p. 75), que les auteurs envisagent comme un cadre juridique (pp. 75-77) permettant aux forces de sécurité d'intervenir sur le territoire national alors que la finalité du renseignement extérieur est uniquement le décideur politique ou militaire.

Pourtant écrit par deux spécialistes du genre, de ce plaidoyer en faveur du « renseignement criminel », il ressort un texte mal ciblé. II est trop partiel pour les spécialistes du renseignement, quel que soit leur genre, et trop spécialisé pour un grand public largement ignorant des subtilités du genre. D'autant que, ça et là, apparaissent des allusions au débat public des années Nicolas Sarkozy, comme le « décèlement précoce », un autre concept sorti de la boîte à outil de la « criminologie » signalé comme venant de Xavier Rauffer (p. 53, note 17). En réalité, comme beaucoup de livre du même genre, il reste un livre daté, difficilement utilisable pour le chercheur.

Gérald Arboit

CRULH, université de Lorraine, F-54000 gerald.arboit@aliceadsl.fr

\section{Frédéric FoREST, dir., Les universités en France. Fonctionnement et enjeux.}

Mont-Saint-Aignan, Publications des universités de Rouen et du Havre, 2012,296 p.

L'ouvrage collectif ressemble à son sujet, ce qui ne saurait surprendre dans la mesure où ses auteurs ont tous en commun une expérience administrative significative à l'Université. La moitié des seize administrateurs sont issus du professorat, quatre étant présidents d'université. II aurait pu être intéressant d'intégrer des non-administrateurs, des étudiants (sur leur pratique de l'institution), voire des entrepreneurs ou des consultants (sur leurs interactions avec les universités, le marché de l'emploi, les structures de gouvernance) ou des figures représentatives des grandes écoles. Aussi fallait-il donner la même place (une contribution) à des catégories comme, d'une part, la recherche et les finances et, d'autre part, le patrimoine immobilier, la politique documentaire ou les ressources humaines? On peut en douter. En outre, on reste sur sa faim aussi bien en matière de finances que de recherche. Le lecteur pourra être rebuté par le code administratif qui prévaut dans ce champ, par ce qu'il verra parfois comme de la langue de bois, et par de nombreuses répétitions ; il est invité à préalablement lire la liste des sigles (pp. 285-290) et prendra connaissance avec profit des tableaux qui présentent des données quantitatives fort utiles. Avant tout, l'ouvrage est une synthèse descriptive et ne fait pratiquement aucune place au débat, ou ne s'engage que rarement. II s'agit d'un livre sage ou, comme l'annonce la quatrième de couverture, d'un état des lieux. Mais, si l'on s'attelle à lire sous le code, parfois le simple déplacement d'un mot ou l'ordre des arguments peut signifier un pas de côté, voire une prise de position. Cependant, l'épilogue de l'éditeur oriente la synthèse vers quelques perspectives d'avenir.

L'introduction de Frédéric Forest (pp. 9-10) présente le livre comme un instrument pour des réformes nécessaires. Sous la plume de Christine Musselin, le premier chapitre (pp. 13-25) décrit l'histoire des universités depuis 1789 (mentionnant la suppression des universités sous la Révolution française) jusqu'à aujourd'hui, en insistant sur quelques points significatifs : le fait que les élites ne sont le plus souvent pas formées dans les universités, l'absence de décision qui caractérise ces dernières, la logique disciplinaire qui entrave - moins aujourd'hui - le processus d'autonomisation, la loi Edgar Faure de 1968 (en avance sur son temps), la Loi relative aux libertés et responsabilité des universités (LRU) et son impact, et les politiques actuelles d'excellence. Dans le second chapitre (pp. 27-45), Éric Piozin décrit la complexité de l'environnement institutionnel, la diversité et les disparités du réseau (voire du maquis) formé par les établissements, et les tentatives de rapprochement et de pilotage. L'auteur rappelle certains faits : deux tiers des étudiants seulement sont à l'Université, les publications sont en déclin. Parmi les faits plus positifs, le texte note la contribution de la mastérisation au rapprochement entre universités et (grandes) écoles, la constitution des pôles de recherche et d'enseignement supérieur (PRES), les fusions récentes (telles en Alsace et en Lorraine), la contractualisation (inaugurée dès 1984 